\title{
THE GENERATOR PROBLEM FOR $\mathcal{Z}$-STABLE $C^{*}$-ALGEBRAS
}

\author{
HANNES THIEL AND WILHELM WINTER
}

\begin{abstract}
The generator problem was posed by Kadison in 1967, and it remains open today. We provide a solution for the class of $C^{*}$-algebras absorbing the Jiang-Su algebra $\mathcal{Z}$ tensorially. More precisely, we show that every unital, separable, $\mathcal{Z}$-stable $C^{*}$-algebra $A$ is singly generated, which means that there exists an element $x \in A$ that is not contained in any proper sub- $C^{*}$-algebra of A.

To give applications of our result, we observe that $\mathcal{Z}$ can be embedded into the reduced group $C^{*}$-algebra of a discrete group that contains a non-cyclic, free subgroup. It follows that certain tensor products with reduced group $C^{*}$-algebras are singly generated. In particular, $C_{r}^{*}\left(F_{\infty}\right) \otimes C_{r}^{*}\left(F_{\infty}\right)$ is singly generated.
\end{abstract}

\section{INTRODUCTION}

By an operator algebra we mean a ${ }^{*}$-subalgebra of $B(H)$ that is either closed in the norm topology (a concrete $C^{*}$-algebra) or the weak operator topology (a von Neumann algebra). One way of realizing an operator algebra is to take a subset of $B(H)$ and consider the smallest operator algebra containing it.

In a trivial way, every operator algebra can be obtained this way. The situation becomes interesting if one imposes restrictions on the generating set, and one natural possibility is to require that it consists of only one element, i.e., to consider operator algebras that are generated by a single operator. It is an old problem to determine which operator algebras arise this way.

More generally, one tries to compute the minimal number of elements that generate a given operator algebra; see Section 2.1 It is often convenient to consider self-adjoint generators. Note that two self-adjoint elements $a, b$ generate the same operator algebra as the element $a+i b$. Thus, if we ask whether an operator algebra is singly generated, it is equivalent to ask whether it is generated by two self-adjoint elements.

In the case of von Neumann algebras, the generator problem was included in Kadison's famous 'Problems on von Neumann algebras', Kad67. This problem list has turned out to be very influential, yet its original form remains unpublished.

Received by the editors April 18, 2012.

2010 Mathematics Subject Classification. Primary 46L05, 46L85; Secondary 46L35.

Key words and phrases. $C^{*}$-algebras, generator problem, single generation, $\mathcal{Z}$-stability.

This research was partially supported by the Centre de Recerca Matemàtica, Barcelona, and the DFG through SFB 878.

The first author was partially supported by the Danish National Research Foundation through the Centre for Symmetry and Deformation, Copenhagen.

The second author was partially supported by EPSRC Grants EP/G014019/1 and $\mathrm{EP} / \mathrm{I} 019227 / 1$. 
It is indirectly available in an article by Ge, Ge03, where a brief summary of the developments around Kadison's famous problems is given.

Question 1.1 (Kadison, Kad67, Problem 14], see also Ge03]). Is every separablyacting 11 von Neumann algebra singly generated?

As noted in She09, there exist singly generated von Neumann algebras that are not separably-acting. However, the separably-acting von Neumann algebras are the natural class for which one might expect single generation. The answer to Question 1.1 is still open in general, but many authors have contributed to show that large classes of separably-acting von Neumann algebras are singly generated.

We just mention an incomplete list of results. It starts with von Neumann, vN31, who showed that the abelian operator algebras named after him are generated by a single self-adjoint element, thus implicitly raising the generator problem. Some thirty years later, this was extended by Pearcy, Pea62, who showed that all von Neumann algebras of type I are singly generated. Then Wogen, Wog69, Theorem 2], proved that all properly infinite von Neumann algebras are singly generated, thus reducing the generator problem to the type $\mathrm{II}_{1}$ case.

Later, this was further reduced to the case of a $\mathrm{II}_{1}$-factor by Willig, Wil74, and then to the case of a finitely generated $\mathrm{II}_{1}$-factor by Sherman, She09, Theorem 3.8]. This means that Question 1.1 has a positive answer if every separably-acting, finitely generated $\mathrm{II}_{1}$-factor is singly generated.

There are many properties known to imply that a $\mathrm{II}_{1}$-factor is singly generated. We just mention that Ge and Popa, GP98, Theorem 6.2], show that every tensorially non-prime $2 \mathrm{II}_{1}$-factor is singly generated. Our main result, Theorem 3.5, can be considered as a partial $C^{*}$-algebraic analog of this result.

Let us also mention that the free group factors $W^{*}\left(F_{k}\right)$ are the outstanding examples of separably-acting von Neumann algebra for which it is not known whether they are singly generated.

In the case of $C^{*}$-algebras, the generator problem is more subtle. There is already no obvious class of $C^{*}$-algebras for which one conjectures that they are singly generated. Every singly generated $C^{*}$-algebra is separable 3 However, the converse is false, and counterexamples can be found among the commutative $C^{*}$ algebras.

In fact, the $C^{*}$-algebra $C_{0}(X)$ is generated by $n$ self-adjoint elements if and only if $X$ can be embedded into $\mathbb{R}^{n}$. Thus, $C_{0}(X)$ is singly generated if and only if $X$ is planar, i.e., can be embedded into the plane $\mathbb{R}^{2}$.

It is easy to see that a $C^{*}$-algebra $A$ is generated by $n$ self-adjoint elements if and only if its minimal unitization $\widetilde{A}$ is generated by $n$ self-adjoint elements. Therefore, we will mostly consider the generator problem for separable, unital $C^{*}$-algebra. In that case, taking the tensor product with a matrix algebra has the effect of reducing the necessary number of generators. If $A$ is generated by $n^{2}+1$ self-adjoint elements, then $A \otimes M_{n}$ is singly generated; see e.g. [Nag04, Theorem 3].

One derives the principle that a $C^{*}$-algebra needs less generators if it is 'more non-commutative'. Consequently, one might expect a (separable) $C^{*}$-algebra to be

\footnotetext{
${ }^{1}$ A von Neumann algebra is called 'separably-acting', or just 'separable', if it is a subalgebra of $B\left(l^{2} \mathbb{N}\right)$, or equivalently if it has a separable predual.

${ }^{2} \mathrm{~A} \mathrm{II}_{1}$-factor $M$ is called tensorially non-prime if it is isomorphic to a tensor product, $M_{1} \bar{\otimes} M_{2}$, of two $\mathrm{II}_{1}$-factors $M_{1}, M_{2}$.

${ }^{3} \mathrm{~A} C^{*}$-algebra is called 'separable' if it contains a countable, norm-dense subset.
} 
singly generated if it is 'maximally non-commutative'. As a non-unital instance of this principle, we note that the stabilization, $A \otimes \mathbb{K}$, of a separable unital $C^{*}$ algebra $A$ is singly generated, OZ76, Theorem 8]. In the unital case, there are at least three natural cases when one considers a $C^{*}$-algebra $A$ to be 'maximally non-commutative', which are the following:

(1) $A$ contains a simple, unital, non-elementary sub- $C^{*}$-algebra,

(2) $A$ contains a sequence of pairwise orthogonal, full elements,

(3) $A$ has no finite-dimensional irreducible representations.

In general, the implications $(1) \Rightarrow(2) \Rightarrow(3)$ hold; it is not known if the converses are true.

Conditions (2) and (3) can also be considered for possibly non-unital $C^{*}$-algebras, and we let $\left(2^{*}\right)$ be the weaker statement that $A$ contains two orthogonal, full elements. The implication ' $(3) \Rightarrow(2)$ ' holds exactly if the implication ' $(3) \Rightarrow\left(2^{*}\right)$ ' holds.

The Global Glimm halving problem asks the following: Given a (possibly nonunital) $C^{*}$-algebra $A$ that satisfies condition (3), does there exist a full map from the cone over $M_{2}$ to $A$ ? It is not known whether the Global Glimm halving problem has a positive answer, but if it does, then it shows that implication ' $(3) \Rightarrow(2)$ ' holds, since the cone over $M_{2}$ contains two orthogonal, full elements.

Let us remark that the analogs of conditions (1) - (3) for von Neumann algebras are all equivalent. In fact, if a von Neumann algebra $M$ has no finite-dimensional representations, then the hyperfinite $\mathrm{II}_{1}$-factor $\mathcal{R}$ unitally embeds into $M$.

Historically, the generator problem for $C^{*}$-algebras is mostly asked for $C^{*}$-algebras that are simple or more generally have no finite-dimensional representations:

Question 1.2. Is every simple, separable, unital $C^{*}$-algebra singly generated?

Question 1.3. Is a separable, unital $C^{*}$-algebra singly generated provided it has no finite-dimensional irreducible representations?

The answers to both questions are open. A positive answer to Question 1.3 implies a positive answer to Question 1.2 of course. The converse is not clear.

Let us mention some results that solve the generator problem for particular classes of separable $C^{*}$-algebras. It was shown by Topping, Top68, that every UHF-algebra is singly generated. This was generalized by Olsen and Zame, OZ76. Theorem 9], who showed that the tensor product, $A \otimes B$, of any separable, unital $C^{*}$-algebra $A$ with a UHF-algebra $B$ is singly generated.

Later, it was shown by Li and Shen, [LS10, Theorem 3.1], that every unital, approximately divisible $C^{*}$-algebra is singly generated. This generalizes the result of Olsen and Zame, since the tensor product with a UHF-algebra is always approximately divisible.

In this article we prove that every separable, unital, $\mathcal{Z}$-stable $C^{*}$-algebra is singly generated; see Theorem 3.8. This generalizes the result of Li and Shen, since every approximately divisible $C^{*}$-algebra is $\mathcal{Z}$-stable; see [TW08, Theorem 2.3]. The notion of $\mathcal{Z}$-stability has proven to be very important in the classification program of nuclear $C^{*}$-algebras (see e.g. Win07] or [ET08]), and it is has been shown that many nuclear, simple $C^{*}$-algebras are $\mathcal{Z}$-stable (see e.g. Win12]). $\mathcal{Z}$-stability is

\footnotetext{
${ }^{4} \mathrm{~A}$ unital $C^{*}$-algebra $A$ is 'approximately divisible' if for every $\varepsilon>0$ and finite subset $F \subset A$ there exists a finite-dimensional, unital sub- $C^{*}$-algebra $B \subset A$ such that $B$ has no characters and $\|x b-b x\| \leq \varepsilon\|b\|$ for all $x \in F, b \in B$.
} 
also relevant in the non-nuclear context; for example, unital $\mathcal{Z}$-stable $C^{*}$-algebras satisfy Kadison's similarity property (see [JW11]).

Let us indicate a few applications of single generation and consequences of a positive answer to the generator problem.

First, it is often easy to prove 'local-to-global' results for maps whose domain is a singly generated operator algebra, where the meaning of 'local' is that the map has a certain property when considered on each element individually. For instance, Sherman studied locally inner 5 automorphisms of operator algebras, She10, and he shows that every such automorphism on a separably-acting von Neumann algebra is inner. Since every locally inner automorphism is easily seen to be inner on a singly generated operator algebra, a positive answer to the generator problem for von Neumann algebras would provide a quick proof of Sherman's result.

Another interest into the generator problem comes from descriptive set theory, which was successfully used in the last few years to study the complexity of classification problems of certain classes of operator algebras. We refer the reader to FTT11 and the references therein. To apply the methods of descriptive set theory one needs to parametrize the class of studied objects.

If $H$ is a separable, infinite-dimensional Hilbert space, then the Borel structure coming from the weak topology makes $B(H)$ into a standard Borel space. This provides a natural parametrization of singly generated operator algebras by assigning to every operator $x \in B(H)$ the operator algebra it generates in $B(H)$. (One may parametrize general countably generated algebras by considering the Borel space of sequences in $B(H)$.)

Lastly, let us mention that it was shown in DSSW08 that the generator problem is connected to the famous free factor problem, which asks whether the free group factors $W^{*}\left(F_{k}\right)$ are isomorphic for different $k$. More precisely, the following dichotomy was shown: Either all free group factors are singly generated, or $W^{*}\left(F_{k}\right)$ is not singly generated for some $k$ (which then implies that the free group factors are pairwise non-isomorphic). So far, it is not known which of the two possibilities occurs.

This paper proceeds as follows:

In Section 2, we set up our notation and give some basic facts about the minimal number of self-adjoint generators (see Subsection 2.1) and $C_{0}(X)$-algebras (see Subsection 2.5).

Section 3 contains the proof of our main result, which states that the tensor product $A \otimes_{\max } B$ of two separable, unital $C^{*}$-algebras is singly generated, if $A$ satisfies condition (2) from above (e.g. $A$ is simple and non-elementary) and $B$ admits a unital embedding of the Jiang-Su algebra $\mathcal{Z}$; see Theorem 3.5

We derive that every separable, unital, $\mathcal{Z}$-stable $C^{*}$-algebra is singly generated; see Theorem 3.8. Our main result can be considered as a (partial) $C^{*}$-algebraic analog of a theorem of Ge and Popa, GP98, Theorem 6.2], which shows that a tensor product, $M \bar{\otimes} N$, of two $\mathrm{II}_{1}$-factors $M, N$ is singly generated. In fact, we can reprove their theorem with our methods; see Corollary 3.12.

In Section 4, we give further applications of our main theorem to tensor products with reduced group $C^{*}$-algebras. We first observe that $\mathcal{Z}$ embeds unitally into

\footnotetext{
${ }^{5}$ An automorphism $\alpha$ of a (unital) operator algebra $A$ is called locally inner if for each element it agrees with an inner automorphism, i.e., if for every element $a \in A$ there exists a unitary $u_{a}$ such that $\alpha(a)=u_{a} a u_{a}^{*}$.
} 
$C_{r}^{*}\left(F_{\infty}\right)$, the reduced group $C^{*}$-algebra of the free group on infinitely many generators; see Subsection 4.1. Consequently, if a discrete group $\Gamma$ contains a non-cyclic free subgroup, then $\mathcal{Z}$ embeds unitally into $C_{r}^{*}(\Gamma)$; see Proposition 4.2.

We deduce that tensor products of the form $A \otimes_{\max } C_{r}^{*}(\Gamma)$ are singly generated if $A$ is a separable, unital $C^{*}$-algebra satisfying condition (2) from above, and $\Gamma$ is a group containing a non-cyclic free subgroup; see Corollary 4.4. For example, $C_{r}^{*}\left(F_{\infty}\right) \otimes C_{r}^{*}\left(F_{\infty}\right)$ is singly generated, although this $C^{*}$-algebra is not $\mathcal{Z}$-stable; see Example 4.5.

\section{Preliminaries}

By a morphism between $C^{*}$-algebras we mean a *-homomorphism, and by an ideal of a $C^{*}$-algebra we understand a closed, two-sided ideal. If $A$ is a $C^{*}$-algebra, then we denote by $\widetilde{A}$ its minimal unitization. Often, we write $M_{k}$ for the $C^{*}$-algebra of $k$-by- $k$ matrices $M_{k}(\mathbb{C})$.

2.1. Let $A$ be a $C^{*}$-algebra, and $A_{\text {sa }} \subset A$ the subset of self-adjoint elements. We say that a set $S \subset A_{\text {sa }}$ generates $A$, denoted $A=C^{*}(S)$, if the smallest sub- $C^{*}$-algebra of $A$ containing $S$ is $A$ itself. We denote by gen $(A)$ the minimal number of self-adjoint generators, i.e., gen $(A)$ the smallest number $n \in\{1,2,3, \ldots, \infty\}$ such that $A$ contains a generating subset $S \subset A_{\text {sa }}$ of cardinality $n$.

We stress that for the definition of $\operatorname{gen}(A)$, the generators are assumed to be self-adjoint. Two self-adjoint elements $a, b$ generate the same $C^{*}$-algebra as the (non-self-adjoint) element $a+i b$. Therefore, a $C^{*}$-algebra $A$ is said to be singly generated if gen $(A) \leq 2$.

For more details on the minimal number of self-adjoint generators we refer the reader to Nagisa, Nag04, where the following facts are also noted for $C^{*}$-algebras $A$ and $B$ :

(1) $\operatorname{gen}(\widetilde{A})=\operatorname{gen}(A)$,

(2) $\operatorname{gen}\left(C^{*}(A, B)\right) \leq \operatorname{gen}(A)+\operatorname{gen}(B)$, if $A, B$ are sub- $C^{*}$-algebras of a common $C^{*}$-algebra, and where $C^{*}(A, B)$ denotes the sub- $C^{*}$-algebra they generate together,

(3) $\operatorname{gen}(A \oplus B)=\max \{\operatorname{gen}(A) \operatorname{gen}(B)\}$ if at least one of the algebras is unital,

(4) $\operatorname{gen}\left(A \otimes M_{n}\right) \leq\left\lceil\frac{\operatorname{gen}(A)-1}{n^{2}}\right\rceil$, if $A$ is unital and $n \in \mathbb{N}$, and where $\lceil t\rceil$ denotes the smallest natural number that is larger than or equal to $t \in \mathbb{R}$.

Let $I \triangleleft A$ be an ideal in a $C^{*}$-algebra $A$. It is easy to see that the minimal number of self-adjoint generators of $A$ dominates that of the quotient $A / I$, i.e., $\operatorname{gen}(A / I) \leq \operatorname{gen}(A)$, and the minimal number of self-adjoint generators of $A$ can be estimated as $\operatorname{gen}(A) \leq \operatorname{gen}(I)+\operatorname{gen}(A / I)$. The following result gives an estimate for gen $(I)$, and it is probably well-known to experts; since we could not locate it in the literature, we include a short proof.

Proposition 2.2. Let $A$ be a $C^{*}$-algebra, and let $I \triangleleft A$ be an ideal. Then $\operatorname{gen}(I) \leq$ $\operatorname{gen}(A)+1$.

Proof. We may assume gen $(A)$ is finite. So let $a_{1}, \ldots, a_{k}$ be a set of self-adjoint generators for $A$. Then $A$ and $I$ are separable, and so $I$ contains a strictly positive element $h$. It follows that $C^{*}(h)$ contains a quasi-central approximate unit; see [AP77, Corollary 3.3] and Arv77]. It is straightforward to show that $I$ is generated by the $k+1$ elements $h, h a_{1} h, \ldots, h a_{k} h$. 
Remark 2.3. Let $A$ be a $C^{*}$-algebra, and $I \triangleleft A$ an ideal. By the above result, $\operatorname{gen}(I) \leq \operatorname{gen}(A)+1$, and in general this is the best possible estimate. For example, if $A=C_{0}([0,1))$ and $I=C_{0}((0,1))$, then $\operatorname{gen}(A)=1$ and $\operatorname{gen}(I)=2$. More generally, examples with $\operatorname{gen}(A)=k$ and $\operatorname{gen}(I)=k+1$ can easily be constructed for every $k$.

The following result is attributed to Kirchberg in Nag04.

Theorem 2.4 (Kirchberg). Every separable, unital, properly infinite $C^{*}$-algebra is singly generated.

Proof. We sketch a proof based on the proof of [OZ76, Theorem 9]. Let $A$ be a separable, unital, properly infinite $C^{*}$-algebra. Then there exist isometries $s_{1}, s_{2}, \ldots \in$ $A$ with pairwise orthogonal ranges (i.e., $A$ contains a unital copy of the Cuntz algebra $\left.\mathcal{O}_{\infty}\right)$.

Let $a_{1}, a_{2}, \ldots \in A$ be a sequence of (positive) generators for $A$ such that their spectra satisfy $\sigma\left(a_{k}\right) \subset\left[1 / 2 \cdot 1 / 4^{k}, 1 / 4^{k}\right]$. A generator for $A$ is given by

$$
x:=\sum_{k \geq 1}\left(s_{k} a_{k} s_{k}^{*}+1 / 2^{k} s_{k}\right) .
$$

As in in the proof of OZ76, Theorem 9], one can show that the spectrum of $x$ satisfies

$$
\sigma(x) \subset\{0\} \cup \bigcup_{k \geq 1}\left[1 / 2 \cdot 1 / 4^{k}, 1 / 4^{k}\right] .
$$

Let $B:=C^{*}(x) \subset A$. Proceeding inductively, one shows that $a_{k}, s_{k} \in B$. We only sketch this for $k=1$. Set $p:=s_{1} s_{1}^{*}$. Let $f_{n}$ be a sequence of polynomials converging uniformly to 1 on $[1 / 8,1 / 4]$ and to 0 on $[0,1 / 16]$. Then $f_{n}(x)$ converges to an element $y \in B$ of the form $y=p+p b(1-p)$ for some $b \in A$. We compute $y y^{*}=p\left(1_{A}+b(1-p) b^{*}\right) p$. Then for a continuous function $f: \mathbb{R} \rightarrow \mathbb{R}$ with $f(0)=0$ and $f(t)=1$ for $t \geq 1$, we get $f\left(y y^{*}\right)=p \in B$. Then $s_{1} a_{1} s_{1}^{*}=p x p \in B$ and $s_{1}=2 \cdot p x(1-p) \in B$, and then also $a_{1} \in B$.

2.5. Let $X$ be a locally compact, $\sigma$-compact, Hausdorff space. A $C_{0}(X)$-algebra is a $C^{*}$-algebra $A$ together with a morphism $\eta: C_{0}(X) \rightarrow Z(M(A))$, from the commutative $C^{*}$-algebra $C_{0}(X)$ to the center of the multiplier algebra of $A$, such that for any approximate unit $\left(u_{\lambda}\right)_{\Lambda}$ of $C_{0}(X), \eta\left(u_{\lambda}\right) a \rightarrow a$ for any $a \in A$, or equivalently, the closure of $\eta\left(C_{0}(X)\right) A$ is all of $A$. Thus, if $X$ is compact, then $\eta$ is necessarily unital. We will usually suppress reference to the structure map, and simply write $f a$ or $f \cdot a$ instead of $\eta(f) a$ for the product of a function $f \in C_{0}(X)$ and an element $a \in A$.

Let $Y \subset X$ be a closed subset, and $U:=X \backslash Y$ its complement (an open subset). Then $C_{0}(U) \cdot A$ is an ideal of $A$, denoted by $A(U)$. The quotient $A / A(U)$ is denoted by $A(Y)$.

Given a point $x \in X$, we write $A(x)$ for $A(\{x\})$, and we call this $C^{*}$-algebra the fiber of $A$ at $x$. For an element $a \in A$, we denote by $a(x)$ the image of $a$ in the fiber $A(x)$. For each $a \in A$, we may consider the map $\check{a}: x \mapsto\|a(x)\|$. This is a real-valued, upper-semicontinuous function on $X$, vanishing at infinity. The $C_{0}(X)$-algebra $A$ is called continuous if $\check{a}$ is a continuous function for each $a \in A$.

For more information on $C_{0}(X)$-algebras we refer the reader to [Kas88, §1] or the more recent [Dad09, $\S 2]$. 
2.6. The Jiang-Su algebra $\mathcal{Z}$ was constructed in [JS99]; it may be regarded as a $C^{*}$ algebraic analog of the hyperfinite $\mathrm{II}_{1}$-factor. It can be obtained as an inductive limit of prime dimension drop algebras $\mathcal{Z}_{p, q}:=\left\{f:[0,1] \rightarrow M_{p} \otimes M_{q} \mid f(0) \in\right.$ $\left.1_{p} \otimes M_{q}, f(1) \in M_{p} \otimes 1_{q}\right\}$.

For more details, we refer the reader to Win11, where $\mathcal{Z}$ is characterized in an entirely abstract manner, and to Rør04 and [RW10, where it is shown that the generalized dimension drop algebra $\mathcal{Z}_{2^{\infty}, 3^{\infty}}:=\left\{f:[0,1] \rightarrow M_{2^{\infty}} \otimes M_{3^{\infty}} \mid f(0) \in\right.$ $\left.1 \otimes M_{3^{\infty}}, f(1) \in M_{2^{\infty}} \otimes 1\right\}$ embeds unitally into $\mathcal{Z}$; in fact, $\mathcal{Z}$ can be written as a stationary inductive limit of $\mathcal{Z}_{2^{\infty}, 3^{\infty}}$.

\section{Results}

Lemma 3.1. Let $A$ be a separable, unital $C^{*}$-algebra. Then $\operatorname{gen}\left(A \otimes \mathcal{Z}_{2^{\infty}, 3^{\infty}}\right) \leq 5$.

Proof. Consider the ideal $I:=A \otimes C_{0}(0,1) \otimes M_{6^{\infty}}$ in $B:=A \otimes \mathcal{Z}_{2^{\infty}, 3^{\infty}}$. The quotient $B / I$ is isomorphic to $\left(A \otimes M_{2^{\infty}}\right) \oplus\left(A \otimes M_{3 \infty}\right)$. Thus, we have a short exact sequence:

$$
A \otimes C_{0}(0,1) \otimes M_{6^{\infty}} \longrightarrow A \otimes \mathcal{Z}_{2^{\infty}, 3^{\infty}} \longrightarrow\left(A \otimes M_{2^{\infty}}\right) \oplus\left(A \otimes M_{3^{\infty}}\right) \cdot
$$

It follows from [OZ76] that the tensor product of a unital, separable $C^{*}$-algebra with a UHF-algebra is singly generated. In particular, $\operatorname{gen}\left(A \otimes M_{2}\right)$, $\operatorname{gen}\left(A \otimes M_{3}\right) \leq 2$. Thus, using the results stated in Subsection 2.1, the quotient satisfies

$$
\operatorname{gen}(B / I)=\max \left\{\operatorname{gen}\left(A \otimes M_{2 \infty}\right), \operatorname{gen}\left(A \otimes M_{3 \infty}\right)\right\} \leq 2 .
$$

Note that $I$ is an ideal in the $C^{*}$-algebra $C:=A \otimes C\left(S^{1}\right) \otimes M_{2}$. We have $\operatorname{gen}(C) \leq 2$, and then $\operatorname{gen}(I) \leq \operatorname{gen}(C)+1 \leq 3$, by Proposition 2.2 Then, the extension is generated by at most $2+3=5$ self-adjoint elements.

The following Lemma 3.2 is a Stone-Weierstrass type result for $C_{0}(X)$-algebras. We prove it using the factorial Stone-Weierstrass conjecture, which was shown for separable $C^{*}$-algebras independently by Longo, Lon84, and Popa, Pop84.

Given a $C^{*}$-algebra $A$, we denote the space of factorial states on $A$ by $F(A)$. If $A$ is unital, then the factorial Stone-Weierstrass conjecture states that a sub- $C^{*}$ algebra $B \subset A$ exhausts $A$ if it contains the unit of $A$ and separates $F(A)$. If $A$ is non-unital, then the conjecture states that a sub- $C^{*}$-algebra $B \subset A$ exhausts $A$ if it separates $F(A) \cup\{0\}$.

See Subsection 2.5 for a short introduction to $C_{0}(X)$-algebras.

Lemma 3.2. Let $A$ be a separable $C_{0}(X)$-algebra, and let $B \subset A$ be a sub- $C^{*}$-algebra such that the following two conditions are satisfied:

(i) For each $x \in X, B$ exhausts the fiber $A(x)$,

(ii) $B$ separates the points of $X$ by full elements, i.e., for each pair of distinct points $x_{0}, x_{1} \in X$ there exists some $b \in B$ such that $b\left(x_{1}\right)$ is full in $A\left(x_{1}\right)$ and $b\left(x_{0}\right)=0$.

Then $A=B$.

Condition (ii) is for instance satisfied if $B$ contains the image of the structure map $\eta: C_{0}(X) \rightarrow Z(M(A))$. 
Proof. Set $Y:=\operatorname{Prim}(Z(M(A)))$, and identify $Z(M(A))$ with $C(Y)$. Let $\pi: A \rightarrow$ $B(H)$ be a non-degenerate factor representation. Then $\pi$ extends to a representation $\tilde{\pi}: M(A) \rightarrow B(H)$. It is straightforward to show $\pi(A)^{\prime \prime}=\tilde{\pi}(M(A))^{\prime \prime}$, so that $\tilde{\pi}$ is a factor representation of $M(A)$. For any $c \in Z(M(A))$, we have $c \in \pi(A)^{\prime} \cap \tilde{\pi}(M(A))^{\prime \prime}=\mathbb{C} \cdot 1_{H}$. Thus, there exists a point $y \in Y$ such that $\tilde{\pi}(c)=c(y) \cdot 1_{H}$ for all $c \in Z(M(A))$. Since $\eta\left(C_{0}(X)\right)$ contains an approximate unit for $A$, we have that $\tilde{\pi} \circ \eta$ is non-zero. Thus, there exists a point $x \in X$ such that $\tilde{\pi} \circ \eta(f)=f(x) \cdot 1_{H}$ for all $f \in C_{0}(X)$. This means that $\tilde{\pi} \circ \eta$ vanishes on the ideal $A(X \backslash\{x\})$, so that $\pi$ factors through the fiber $A(x)$.

It follows easily from condition (i) that $B$ separates $\{0\}$ and $F(A)$. To show that $B$ separates $F(A)$, let $\varphi_{1}, \varphi_{2}$ be two different, non-degenerate factor states of $A$. We have shown above that there are two points $x_{1}, x_{2} \in X$ such that $\varphi_{i}$ factors through $A\left(x_{i}\right)$, and we denote by $\bar{\varphi}_{i}: A\left(x_{i}\right) \rightarrow \mathbb{C}$ the induced factor state on $A\left(x_{i}\right)$, for $i=1,2$. We distinguish two cases:

Case 1: $x_{1}=x_{2}$. In this case, since $\varphi_{1} \neq \varphi_{2}$, there exists an element $a \in A$ such that $\varphi_{1}(a) \neq \varphi_{2}(a)$. By condition (i), there exists some element $b \in B$ such that $b\left(x_{1}\right)=a\left(x_{1}\right)$. Note that for $i=1,2$ we have

$$
\varphi_{i}(b)=\bar{\varphi}_{i}\left(b\left(x_{1}\right)\right)=\bar{\varphi}_{i}\left(a\left(x_{1}\right)\right)=\varphi_{i}(a) .
$$

Thus, $b$ separates the two states.

Case 2: $x_{1} \neq x_{2}$. In this case, by condition (ii), there exists an element $b \in B$ such that $b\left(x_{2}\right)$ is full in $A\left(x_{2}\right)$ and $b\left(x_{1}\right)=0$. Since $\varphi_{2} \neq 0$, there exists an element $a \in A$ such that $\left|\varphi_{2}(a)\right|=\left|\bar{\varphi}_{2}\left(a\left(x_{2}\right)\right)\right| \geq 1$.

Since $b\left(x_{2}\right)$ is full, there exist finitely many elements $g_{i}, h_{i} \in A\left(x_{2}\right)$ such that $\left\|a\left(x_{2}\right)-\sum_{i} c_{i} b\left(x_{2}\right) d_{i}\right\|<1$. By condition (i), there exist elements $\tilde{g}_{i}, \tilde{h}_{i} \in B$ such that $\tilde{g}_{i}\left(x_{2}\right)=g_{i}$ and $\tilde{h}_{i}\left(x_{2}\right)=h_{i}$. Set $b^{\prime}:=\sum_{i} \tilde{c}_{i} b \tilde{d}_{i}$. Then $\left|\varphi_{2}\left(b^{\prime}\right)\right|=\left|\bar{\varphi}_{2}\left(b^{\prime}\left(x_{2}\right)\right)\right|>$ 0 , while $b^{\prime}\left(x_{1}\right)=0$. This shows that $b^{\prime}$ separates the two states.

We have shown that $B$ separates $F(A) \cup\{0\}$, and therefore $B=A$ by the factorial Stone-Weierstrass conjecture.

Lemma 3.3. Let $A$ be a unital $C^{*}$-algebra with $\operatorname{gen}(A) \leq 3$. Then there exist a positive element $x \in A \otimes \mathcal{Z}_{2,3}$ and two positive, full elements $y^{\prime}, z^{\prime} \in \mathcal{Z}_{2,3}$ such that $A \otimes \mathcal{Z}_{2,3}$ is generated by $x$ and $1 \otimes y^{\prime}$, and further $y^{\prime}$ and $z^{\prime}$ are orthogonal.

Proof. We consider $\mathcal{Z}_{2,3}$ as the $C^{*}$-algebra of continuous functions from $[0,1]$ to $M_{6}$ with the boundary conditions

$$
f(0)=\left(\begin{array}{ccc}
Y & & \\
& Y & \\
& & Y
\end{array}\right) \quad f(1)=\left(\begin{array}{ll}
Z & \\
& Q Z Q^{*}
\end{array}\right),
$$

where $Y \in M_{2}$ and $Z \in M_{3}$ are arbitrary matrices, and $Q \in M_{3}$ is the following fixed permutation matrix:

$$
Q=\left(\begin{array}{lll} 
& & 1 \\
1 & & \\
& 1 &
\end{array}\right)
$$


This means that $f(0), f(1) \in M_{6}$ have the following form:

$$
f(0)=\left(\begin{array}{cccccc}
\mu_{11} & \mu_{12} & & & & \\
\mu_{21} & \mu_{22} & & & & \\
& & \mu_{11} & \mu_{12} & & \\
& & \mu_{21} & \mu_{22} & & \\
& & & & \mu_{11} & \mu_{12} \\
& & & & \mu_{21} & \mu_{22}
\end{array}\right) f(1)=\left(\begin{array}{llllll}
\lambda_{11} & \lambda_{12} & \lambda_{13} & & & \\
\lambda_{21} & \lambda_{22} & \lambda_{23} & & & \\
\lambda_{31} & \lambda_{22} & \lambda_{33} & & & \\
& & & \lambda_{33} & \lambda_{31} & \lambda_{32} \\
& & & \lambda_{13} & \lambda_{11} & \lambda_{12} \\
& & & \lambda_{23} & \lambda_{21} & \lambda_{22}
\end{array}\right),
$$

for numbers $\mu_{i, j}, \lambda_{i, j} \in \mathbb{C}$.

Note that $\mathcal{Z}_{2,3}$ is naturally a continuous $C([0,1])$-algebra, with fibers $\mathcal{Z}_{2,3}(0) \cong$ $M_{2}, \mathcal{Z}_{2,3}(1) \cong M_{3}$, and $\mathcal{Z}_{2,3}(t) \cong M_{6}$ for points $t \in(0,1) \subset[0,1]$.

Let $a, b, c \in A$ be a set of invertible, positive generators for $A$. Denote by $e_{i, j}$ the matrix units in $M_{6}$. To shorten notation, for indices $i, j$ set $f_{i, j}:=e_{i, j}+e_{j, i}$. For $t \in[0,1]$ we define the following element of $A \otimes M_{6}$ :

$$
\begin{aligned}
x_{t} & :=a \otimes\left(e_{1,1}+(1-t) \cdot e_{3,3}+e_{5,5}\right) \\
& +b \otimes\left(f_{1,2}+(1-t) \cdot f_{3,4}+f_{5,6}\right) \\
& +c \otimes\left(e_{2,2}+(1-t) \cdot e_{4.4}+e_{6,6}\right) \\
& +1_{A} \otimes\left(t \cdot f_{2,3}+t \cdot f_{4,5}+\delta(t) \cdot f_{1,3}\right),
\end{aligned}
$$

where $\delta:[0,1] \rightarrow[0,1]$ is a continuous function on $[0,1]$ that takes the value 0 at the endpoints 0 and 1 , and is strictly positive at each point $t \in(0,1)$, e.g., $\delta$ could be given by $\delta(t)=1 / 4-(t-1 / 2)^{2}$. We also define for $t \in[0,1]$ two elements of $M_{6}$ :

$$
\begin{aligned}
& y_{t}^{\prime}:=e_{1,1}+(1-t) \cdot e_{3,3}+e_{5,5}, \\
& z_{t}^{\prime}:=e_{2,2}+(1-t) \cdot e_{4,4}+e_{6,6} .
\end{aligned}
$$

It is easy to check that the assignment $x: t \mapsto x_{t}$ defines an element $x \in A \otimes \mathcal{Z}_{2.3}$. Similarly, we get two elements $y^{\prime}, z^{\prime} \in \mathcal{Z}_{2.3}$ defined via $t \mapsto y_{t}^{\prime}$ and $t \mapsto z_{t}^{\prime}$. In matrix form, these elements look as follows:

$$
x_{t}:=\left(\begin{array}{cc|cc|cc}
a & b & \delta(t) & & & \\
b & c & t & & \\
\hline \delta(t) & t & (1-t) a & (1-t) b & & \\
& & (1-t) b & (1-t) c & t & \\
\hline & & & t & a & b \\
& & & & b & c
\end{array}\right),
$$

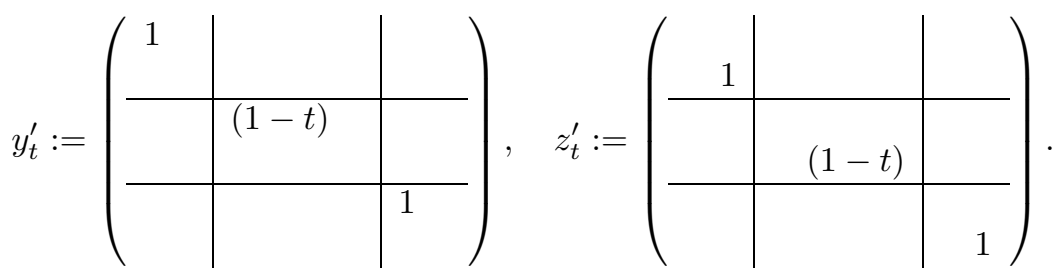

Set $y:=1 \otimes y^{\prime}$, and let $D:=C^{*}(x+1, y)$ be the sub- $C^{*}$-algebra of $E:=A \otimes \mathcal{Z}_{2,3}$ generated by the two self-adjoint elements $x+1$ and $y$. Since $x \geq 0$, we get that both 1 and $x$ lie in $C^{*}(x+1)$. It follows that $D=C^{*}(1, x, y)$, and we will show that $D=E$. Note that $E$ has a natural continuous $C([0,1])$-algebra structure (induced 
by the one of $\left.\mathcal{Z}_{2,3}\right)$, with fibers $E(0) \cong A \otimes M_{2}, E(1) \cong A \otimes M_{3}$, and $E(t) \cong A \otimes M_{6}$ for points $t \in(0,1) \subset[0,1]$.

Let $J:=E((0,1)) \triangleleft E$ be the natural ideal corresponding to the open set $(0,1) \subset$ $[0,1]$. Note that $J \cong A \otimes C_{0}((0,1)) \otimes M_{6}$, and $J$ is naturally a continuous $C_{0}((0,1))$ algebra. We will show in two steps that $D$ exhausts the ideal $J$ (i.e., $D \cap J=J$ ) and the quotient $E / J$ (i.e., $D /(D \cap J)=E / J)$.

Step 1. We want to apply Lemma 3.2 to the $C((0,1))$-algebra $J$ with sub- $C^{*}$-algebra $D \cap J$. To verify condition (ii), note that the $C^{*}$-algebra generated by $y^{\prime}$ contains $C_{0}((0,1)) \otimes e_{3,3}$. Therefore, $D \cap J$ contains $1_{A} \otimes C_{0}((0,1)) \otimes e_{3,3}$, which separates the points of $(0,1)$. Since $1_{A} \otimes e_{3,3} \in E(t) \cong A \otimes M_{6}$ is full, condition (ii) of Lemma 3.2 holds, and it remains to verify condition (i).

We need to show that $D \cap J$ exhausts all fibers of $J$. Fix some $t \in(0,1)$, and set $D_{t}:=C^{*}\left(1, x_{t}, y_{t}\right) \subset A \otimes M_{6}$. To simplify notation, we write $\bar{e}_{i, j}$ for the matrix units $1_{A} \otimes e_{i, j} \in A \otimes M_{6}$. We need to show that $D_{t}$ is all of $A \otimes M_{6}$. This will follow if $D_{t}$ contains all $\bar{e}_{i, j}$, and for this it is enough to show that the off-diagonal matrix units $\bar{e}_{i, i+1}$ are in $D_{t}$, for $i=1, \ldots, 5$.

The spectrum of $y_{t}$ is $\{0,1-t, 1\}$. Applying functional calculus to $y_{t}$ we obtain that the following three elements lie in $D_{t}$ :

$$
\begin{aligned}
u & :=\bar{e}_{1,1}+\bar{e}_{5,5}, \\
v & :=\bar{e}_{3,3}, \\
w & :=1-v-u=\bar{e}_{2,2}+\bar{e}_{4,4}+\bar{e}_{6,6} .
\end{aligned}
$$

Then, we proceed as follows:

1. $\bar{e}_{1,3}=\delta(t)^{-1} u x_{t} v \in D_{t}$, and so $\bar{e}_{1,1}, \bar{e}_{5,5} \in D_{t}$.

2. $g:=b \otimes e_{1,2}=\bar{e}_{1,1} x_{t} w \in D_{t}$. It follows that $b \otimes e_{1,1}=\left(g g^{*}\right)^{1 / 2} \in D_{t} ;$ cf. OZ76. Then $b^{-1} \otimes e_{1,1} \in C^{*}\left(b \otimes e_{1,1}\right) \subset D_{t}$, and so $\bar{e}_{1,2}=\left(b^{-1} \otimes e_{1,1}\right) \cdot g \in D_{t}$ and $\bar{e}_{2,2} \in D_{t}$.

3. $b \otimes e_{3,4}=(1-t)^{-1} \bar{e}_{3,3} x_{t}\left(w-\bar{e}_{2,2}\right) \in D_{t}$. Arguing as above, it follows that $\bar{e}_{3,4} \in D_{t}$, and then $\bar{e}_{4,4}, \bar{e}_{6,6} \in D_{t}$.

4. $\bar{e}_{2,3}=t^{-1} \bar{e}_{2,2} x_{t} \bar{e}_{3,3} \in D_{t}$.

5. $\bar{e}_{4,5}=t^{-1} \bar{e}_{4,4} x_{t} \bar{e}_{5,5} \in D_{t}$.

6. $b \otimes e_{5,6}=\bar{e}_{5,5} x_{t} \bar{e}_{6,6} \in D_{t}$, and so $\bar{e}_{5,6} \in D_{t}$.

This shows that $D \cap J$ exhausts the fibers of $J$. We may apply Lemma 3.2 and deduce $D \cap J=J$, which finishes Step 1 .

Step 2. We want to show that $D / J$ exhausts $E / J=E(\{0,1\}) \cong A \otimes\left(M_{2} \oplus M_{3}\right)$. Let us denote the matrix units in $M_{2}$ by $e_{i, j}^{(0)}, i=1,2$, and the matrix units in $M_{3}$ by $e_{i, j}^{(1)}, i=1,2,3$. To simplify notation, we write $\bar{e}_{i, j}^{(k)}$ for the matrix units $1_{A} \otimes e_{i, j}^{(k)} \in A \otimes\left(M_{2} \oplus M_{3}\right)$. Let us denote the image of $x$ and $y$ in $D / J$ by $v$ and $w$ :

$$
\begin{aligned}
v & =a \otimes\left(e_{1,1}^{(0)}+e_{1,1}^{(1)}\right)+b \otimes\left(e_{1,2}^{(0)}+e_{2,1}^{(0)}+e_{1,2}^{(1)}+e_{2,1}^{(1)}\right)+c \otimes\left(e_{2,2}^{(0)}+e_{2,2}^{(1)}\right)+\bar{e}_{2,3}^{(1)}+\bar{e}_{3,2}^{(1)} \\
& =\left(\begin{array}{ll}
a & b \\
b & c
\end{array}\right) \oplus\left(\begin{array}{lll}
a & b & \\
b & c & 1 \\
& 1 &
\end{array}\right), \\
w & =\bar{e}_{1,1}^{(0)}+\bar{e}_{1,1}^{(1)}=\left(\begin{array}{ll}
1 & 0 \\
0 & 0
\end{array}\right) \oplus\left(\begin{array}{ccc}
1 & & \\
& 0 & \\
& & 0
\end{array}\right) .
\end{aligned}
$$


As in Step 1, it is enough to show that $D / J$ contains the off-diagonal matrix units $\bar{e}_{1,2}^{(0)}, \bar{e}_{1,2}^{(1)}$ and $\bar{e}_{2,3}^{(1)}$. We argue as follows:

1. $g:=w v(1-w)=b \otimes\left(e_{1,2}^{(0)}+e_{1,2}^{(1)}\right) \in D / J$. As in Step 1, it follows that $b \otimes\left(e_{1,1}^{(0)}+e_{1,1}^{(1)}\right)=\left(g g^{*}\right)^{1 / 2} \in D / J$. Then $b^{-1} \otimes\left(e_{1,1}^{(0)}+e_{1,1}^{(1)}\right) \in D / J$, and so $\bar{e}_{1,2}^{(0)}+\bar{e}_{1,2}^{(1)}=\left(b^{-1} \otimes\left(e_{1,1}^{(0)}+e_{1,1}^{(1)}\right)\right) \cdot g \in D / J$. It follows that $\bar{e}_{2,2}^{(0)}+\bar{e}_{2,2}^{(1)} \in D / J$.

2. $\bar{e}_{3,3}^{(1)}=1-w-\left(\bar{e}_{2,2}^{(0)}+\bar{e}_{2,2}^{(1)}\right) \in D / J$.

3. $\bar{e}_{2,3}^{(1)}=v \bar{e}_{3,3}^{(1)} \in D / J$, and so $\bar{e}_{2,2}^{(1)} \in D / J$.

4. $b \otimes e_{1,2}^{(1)}=w v \bar{e}_{2,2}^{(1)} \in D / J$. Again, this implies $\bar{e}_{1,2}^{(1)} \in D / J$, and so $\bar{e}_{1,1}^{(1)} \in D / J$.

5. $\bar{e}_{1,1}^{(0)}=w-\bar{e}_{1,1}^{(1)} \in D / J$.

6. $\bar{e}_{2,2}^{(0)}=1-w-\bar{e}_{2,2}^{(1)}-\bar{e}_{3,3}^{(1)} \in D / J$.

7. $b \otimes e_{1,2}^{(0)}=\bar{e}_{1,1}^{(0)} v \bar{e}_{2,2}^{(0)} \in D / J$. Again, this implies $\bar{e}_{1,2}^{(0)} \in D / J$.

This finishes Step 2.

We have seen that $A \otimes \mathcal{Z}_{2,3}$ is generated by $x+1$ and $y$. Moreover, $z^{\prime}$ is full, positive and orthogonal to $y^{\prime}$.

Lemma 3.4. Let $A$ be a separable, unital $C^{*}$-algebra. Then there exist a positive element $x \in A \otimes \mathcal{Z}_{2^{\infty}, 3^{\infty}}$ and two positive, full elements $y^{\prime}, z^{\prime} \in \mathcal{Z}_{2^{\infty}, 3^{\infty}}$ such that $A \otimes \mathcal{Z}_{2 \infty, 3 \infty}$ is generated by $x$ and $y:=1 \otimes y^{\prime}$, and further $y^{\prime}$ and $z^{\prime}$ are orthogonal.

Proof. Let $B:=A \otimes \mathcal{Z}_{2^{\infty}, 3^{\infty}}$. Note that $\mathcal{Z}_{2^{\infty}, 3^{\infty}} \otimes \mathcal{Z}_{2,3}$ is naturally a $C([0,1] \times[0,1])$ algebra. Then, the quotient corresponding to the diagonal $\{(t, t) \mid t \in[0,1]\} \subset[0,1] \times$ $[0,1]$ is isomorphic to $\mathcal{Z}_{2^{\infty}, 3 \infty}$, and we denote the resulting surjective morphism by $\pi: \mathcal{Z}_{2^{\infty}, 3^{\infty}} \otimes \mathcal{Z}_{2,3} \rightarrow \mathcal{Z}_{2^{\infty}, 3^{\infty}}$. We proceed in two steps.

Step 1. We show that $\operatorname{gen}(B) \leq k+1$ implies $\operatorname{gen}(B) \leq k$ for $k \geq 2$. So assume $B$ is generated by the self-adjoint, invertible elements $a_{1}, \ldots, a_{k+1}$. The sub- $C^{*}$-algebra $C:=C^{*}\left(a_{k-1}, a_{k}, a_{k+1}\right) \subset B$ is unital and satisfies gen $(C) \leq 3$. Consider the $C^{*}$-algebra $B \otimes \mathcal{Z}_{2,3}$. By Lemma 3.3 the sub- $C^{*}$-algebra $C \otimes \mathcal{Z}_{2,3}$ is generated by two self-adjoint elements, say $b, c$.

One readily checks that $B \otimes \mathcal{Z}_{2,3}$ is generated by the $k$ self-adjoint elements $a_{1} \otimes 1, \ldots, a_{k-2} \otimes 1, b, c$. Since $B=A \otimes \mathcal{Z}_{2^{\infty}, 3^{\infty}}$ is isomorphic to a quotient of $B \otimes \mathcal{Z}_{2,3}=A \otimes \mathcal{Z}_{2 \infty, 3 \infty} \otimes \mathcal{Z}_{2,3}$, we obtain $\operatorname{gen}(B) \leq \operatorname{gen}\left(B \otimes \mathcal{Z}_{2,3}\right) \leq k$.

Step 2. By Lemma 3.1, we have $\operatorname{gen}(B) \leq 5$. Applying Step 1 several times, we obtain $\operatorname{gen}(B) \leq 3$.

It follows from Lemma 3.3 that there exists a positive element $\tilde{x} \in B \otimes \mathcal{Z}_{2,3}$ and two positive, full elements $\tilde{y}^{\prime}, \tilde{z}^{\prime} \in \mathcal{Z}_{2,3}$ such that $B \otimes \mathcal{Z}_{2,3}$ is generated by $\tilde{x}$ and $1 \otimes \tilde{y}^{\prime}$, and further $\tilde{y}^{\prime}$ and $\tilde{z}^{\prime}$ are orthogonal.

Consider the surjective morphism id $\otimes \pi: A \otimes \mathcal{Z}_{2^{\infty}, 3^{\infty}} \otimes \mathcal{Z}_{2,3} \rightarrow A \otimes \mathcal{Z}_{2^{\infty}, 3^{\infty}}$. One checks that the elements $x:=(\mathrm{id} \otimes \pi)(\tilde{x}) \in A \otimes \mathcal{Z}_{2^{\infty}, 3^{\infty}}$ and $y^{\prime}:=\pi\left(\tilde{y}^{\prime}\right), z^{\prime}:=$ $\pi\left(\tilde{z}^{\prime}\right) \in \mathcal{Z}_{2^{\infty}, 3^{\infty}}$ have the desired properties.

Theorem 3.5. Let $A, B$ be two separable, unital $C^{*}$-algebras. Assume the following:

(1) A contains a sequence $a_{1}, a_{2}, \ldots$ of full, positive elements that are pairwise orthogonal,

(2) $B$ admits a unital embedding of the Jiang-Su algebra $\mathcal{Z}$. 
Then $A \otimes_{\max } B$ is singly generated. Every other tensor product $A \otimes_{\lambda} B$ is a quotient of $A \otimes_{\max } B$, and therefore is also singly generated.

Proof. There exists a unital embedding of $\mathcal{Z}_{2^{\infty}, 3^{\infty}}$ in $\mathcal{Z}$, so we may assume that there is a unital embedding of $\mathcal{Z}_{2^{\infty}, 3^{\infty}}$ in $B$. We may assume that the elements $a_{1}, a_{2}, \ldots \in A$ are contractive.

Choose a sequence $b_{1}, b_{2}, \ldots \in B$ of contractive, positive elements that is dense in the set of all contractive, positive elements of $B$.

Consider the sub- $C^{*}$-algebra $A \otimes \mathcal{Z}_{2 \infty, 3 \infty} \subset A \otimes_{\max } B$. By Lemma 3.4 there exist a positive element $x \in A \otimes \mathcal{Z}_{2^{\infty}, 3^{\infty}}$ and two full, positive elements $y^{\prime}, z^{\prime} \in \mathcal{Z}_{2^{\infty}, 3^{\infty}}$ such that $A \otimes \mathcal{Z}_{2^{\infty}, 3^{\infty}}$ is generated by $x$ and $y:=1 \otimes y^{\prime}$, and further $y^{\prime}$ and $z^{\prime}$ are orthogonal.

Define the following two elements of $A \otimes_{\max } B$ :

$$
v:=x, \quad w:=1 \otimes y^{\prime}-\sum_{k \geq 1} 1 / 2^{k} \cdot a_{k} \otimes\left(z^{\prime} b_{k} z^{\prime}\right) .
$$

Let $D:=C^{*}(v, w)$ be the sub- $C^{*}$-algebra of $A \otimes_{\max } B$ generated by $v$ and $w$. We claim that $D=A \otimes B$.

Step 1. We show $A \otimes \mathcal{Z}_{2^{\infty}, 3^{\infty}} \subset D$. Note that the two elements $1 \otimes y^{\prime}$ and $\sum_{k \geq 1} 1 / 2^{k}$. $a_{k} \otimes\left(z^{\prime} b_{k} z^{\prime}\right)$ are positive and orthogonal. It follows that $1 \otimes y^{\prime}$ is the positive part of $w$, and therefore $1 \otimes y^{\prime} \in D$. Therefore, $C^{*}\left(v, 1 \otimes y^{\prime}\right)=A \otimes \mathcal{Z}_{2^{\infty}, 3^{\infty}} \subset D$.

Step 2. We show $1 \otimes B \subset D$. We have

$$
g:=\sum_{k \geq 1} 1 / 2^{k} \cdot a_{k} \otimes\left(z^{\prime} b_{k} z^{\prime}\right) \in D .
$$

It follows from Step 1 that $a_{k} \otimes 1 \in D$, and so

$$
a_{k}^{2} \otimes\left(z^{\prime} b_{k} z^{\prime}\right)=2^{k} \cdot\left(a_{k} \otimes 1\right) g \in D .
$$

Since $a_{k}^{2}$ is full, there exist finitely many elements $c_{i}, d_{i} \in A$ such that $1_{A}=$ $\sum_{i} c_{i} a_{k}^{2} d_{i}$. By Step 1 , we have $c_{i} \otimes 1, d_{i} \otimes 1 \in D$. Then

$$
1 \otimes\left(z^{\prime} b_{k} z^{\prime}\right)=\sum_{i}\left(c_{i} \otimes 1\right)\left(a_{k}^{2} \otimes\left(z^{\prime} b_{k} z^{\prime}\right)\right)\left(d_{i} \otimes 1\right) \in D,
$$

for each $k$.

Let $b \in B$ be a contractive, positive element. Then $b=\lim _{j} b_{k(j)}$ for certain indices $k(j)$. Then $1 \otimes\left(z^{\prime} b z^{\prime}\right)=\lim _{j} 1 \otimes\left(z^{\prime} b_{k(j)} z^{\prime}\right) \in D$. It follows that the hereditary sub- $C^{*}$-algebra $1 \otimes \overline{z^{\prime} B z^{\prime}}$ is contained in $D$. Since $z^{\prime}$ is full in $\mathcal{Z}_{2^{\infty}, 3^{\infty}}$, there exist finitely many elements $c_{i}, d_{i} \in \mathcal{Z}_{2^{\infty}, 3^{\infty}}$ such that $1_{B}=\sum_{i} c_{i} z^{\prime} d_{i}$. We have seen that $1 \otimes z^{\prime} b z^{\prime} \in D$ for any $b \in B$. Then

$$
1 \otimes b z^{\prime}=\sum_{i}\left(1 \otimes c_{i}\right)\left(1 \otimes z^{\prime} d_{i} b z^{\prime}\right) \in D
$$

for any $b \in B$. Similarly

$$
1 \otimes b=\sum_{i}\left(1 \otimes b c_{i} z^{\prime}\right)\left(1 \otimes d_{i}\right) \in D
$$

for any $b \in B$, as desired.

It follows from Steps 1 and 2 that for each $a \in A$ and $b \in B$ the simple tensor $a \otimes b$ is contained in $D$. The conclusion follows since $A \otimes_{\max } B$ is the closure of the linear span of simple tensors. 
Corollary 3.6. Let $A, B$ be two separable, unital $C^{*}$-algebras that both admit a unital embedding of the Jiang-Su algebra $\mathcal{Z}$. Then $A \otimes_{\max } B$ is singly generated.

Proof. It is easy to verify that condition (1) of Theorem 3.5 is fulfilled if $A$ admits a unital embedding of $\mathcal{Z}$.

Example 3.7. Consider two unital, separable $C^{*}$-algebras $A$ and $B$ that have real rank zero and no (non-zero) finite-dimensional representations. As shown by Elliott and Rørdam, ER06, the Jiang-Su algebra embeds unitally into $A$ and $B$. Therefore, $A \otimes_{\max } B$ is singly generated by the above Corollary 3.6.

Theorem 3.8. Let $A$ be a unital, separable $C^{*}$-algebra. Then $A \otimes \mathcal{Z}$ is singly generated.

Proof. Note that $A \otimes \mathcal{Z} \cong(A \otimes \mathcal{Z}) \otimes \mathcal{Z}$. It is clear that both $A \otimes \mathcal{Z}$ and $\mathcal{Z}$ admit unital embeddings of $\mathcal{Z}$. Then apply the above Corollary 3.6.

Corollary 3.9. Let $A$ be a separable $C^{*}$-algebra. Then $\operatorname{gen}(A \otimes \mathcal{Z}) \leq 3$.

Proof. Let $\widetilde{A}$ be the minimal unitization of $A$. It follows from Theorem 3.8 that $\operatorname{gen}(\widetilde{A} \otimes \mathcal{Z}) \leq 2$. Since $A \otimes \mathcal{Z}$ is an ideal in $\widetilde{A} \otimes \mathcal{Z}$, we get $\operatorname{gen}(A \otimes \mathcal{Z}) \leq$ $\operatorname{gen}(\widetilde{A} \otimes \mathcal{Z})+1 \leq 3$ from Proposition 2.2 as desired.

Our results allow us to give new proofs for results about the single generation of certain von Neumann algebras.

Proposition 3.10. Assume $M, N$ are separably-acting von Neumann algebras that both admit a unital embedding of the hyperfinite $I I_{1}$-factor. Then $M \bar{\otimes} N$ is singly generated.

Proof. Consider the GNS-representation $\pi: \mathcal{Z} \rightarrow B(H)$ of the Jiang-Su algebra with respect to its tracial state. The weak closure, $\pi(\mathcal{Z})^{\prime \prime}$, is isomorphic to the hyperfinite $\mathrm{II}_{1}$-factor $\mathcal{R}$. Thus, there exists a weakly dense, unital copy of $\mathcal{Z}$ inside $\mathcal{R}$.

Choose weakly dense, separable, unital $C^{*}$-algebras $A_{0} \subset M$, and similarly $B_{0} \subset$ $N$. Consider $\mathcal{Z} \subset \mathcal{R} \subset M$ and set $A:=C^{*}\left(A_{0}, \mathcal{Z}\right) \subset M$. Similarly set $B:=$ $C^{*}\left(B_{0}, \mathcal{Z}\right) \subset N$.

Then $A$ and $B$ are separable, unital $C^{*}$-algebras that both contain unital copies of the Jiang-Su algebra. By Corollary 3.6, $A \otimes_{\max } B$ is singly generated.

Consider the sub- $C^{*}$-algebra $C:=C^{*}(A \bar{\otimes} 1,1 \bar{\otimes} B) \subset M \bar{\otimes} N$. Then $C$ is a quotient of $A \otimes_{\max } B$, and therefore singly generated. Since $C$ is weakly dense in $M \bar{\otimes} N$, we obtain that $M \bar{\otimes} N$ is singly generated, as desired.

Remark 3.11. We note that a von Neumann algebra $M$ admits a unital embedding of $\mathcal{R}$ if and only if $M$ has no (non-zero) finite-dimensional representations.

The analogous statement for $C^{*}$-algebras would be that a $C^{*}$-algebra $A$ admits a unital embedding of $\mathcal{Z}$ if and only if $A$ has no (non-zero) finite-dimensional representations. It was shown by Elliott and Rørdam, [ER06], that this is true for $C^{*}$-algebras of real rank zero. However, in [DHTW09] a simple, separable, unital, non-elementary $\mathrm{AH}$-algebra is constructed into which $\mathcal{Z}$ does not embed.

As a particular case of Proposition 3.10 we obtain the following result of Ge and Popa.

Corollary 3.12 (Ge, Popa, GP98, Theorem 6.2]). Assume $M, N$ are separablyacting $I I_{1}$-factors. Then $M \bar{\otimes} N$ is singly generated. 


\section{Applications}

In this section we show that the Jiang-Su algebra $\mathcal{Z}$ embeds unitally into the reduced group $C^{*}$-algebras, $C_{r}^{*}(\Gamma)$, of groups $\Gamma$ that contain a non-cyclic free subgroup; see Proposition 4.2. We only consider discrete groups, and we let $F_{k}$ denote the free group with $k$ generators $(k \in\{2,3 \ldots, \infty\})$.

We can apply Theorem 3.5 to show that certain tensor products of the form $A \otimes_{\max } C_{r}^{*}(\Gamma)$ are singly generated; see Corollary 4.4 In particular, $C_{r}^{*}\left(F_{\infty}\right) \otimes$ $C_{r}^{*}\left(F_{\infty}\right)$ is singly generated, although it is not $\mathcal{Z}$-stable; see Example 4.5.

4.1. It was shown by Robert, Rob12, that the Jiang-Su algebra $\mathcal{Z}$ embeds unitally into $C_{r}^{*}\left(F_{\infty}\right)$. A key observation is that $C_{r}^{*}\left(F_{\infty}\right)$ has strict comparison of positive elements. This follows from the work of Dykema and Rørdam on reduced free product $C^{*}$-algebras; see DR98 and DR00.

Dykema and Rørdam study the comparison of projections, but this can be generalized to obtain results about the comparison of positive elements, as noted by Robert, [Rob12]. In particular, DR98, Lemma 5.3] and [DR00, Theorem 2.1] can be generalized, and it follows that $C_{r}^{*}\left(F_{\infty}\right)$ has strict comparison of positive elements.

Proposition 4.2. If $\Gamma$ is a discrete group that contains $F_{\infty}$ as a subgroup, then $\mathcal{Z}$ embeds unitally into $C_{r}^{*}(\Gamma)$.

Proof. In general, for any subgroup $\Gamma_{1}$ of a discrete group $\Gamma$, we have a unital embedding $C_{r}^{*}\left(\Gamma_{1}\right) \subset C_{r}^{*}(\Gamma)$. Hence, if $F_{\infty}$ is a subgroup of $\Gamma$, then $C_{r}^{*}(\Gamma)$ contains a unital copy of $C_{r}^{*}\left(F_{\infty}\right)$, which in turn contains a unital copy of $\mathcal{Z}$.

Remark 4.3. Every non-cyclic free group $F_{k}(k \geq 2)$ contains $F_{\infty}$ as a subgroup. In general, by the Nielsen-Schreier theorem, every subgroup of a free group is again free. Thus, if $a, b$ are free elements, then the the elements $a^{k} b^{k}$ generate a subgroup $\Gamma=\left\langle a^{k} b^{k}, k \geq 1\right\rangle$ that is free, and since none of the elements $a^{k} b^{k}$ is contained in the subgroup generated by the other elements, we have $\Gamma \cong F_{\infty}$.

Thus, when we ask which discrete groups contain $F_{\infty}$ as a subgroup, we are equivalently asking which groups $\Gamma$ contain a non-cyclic free subgroup. It is a necessary condition that $\Gamma$ is non-amenable. The converse implication is known as the von Neumann conjecture, but this was disproved in 1980 by Ol'shanskij.

A counterexample is the so-called Tarski monster groups, in which every nontrivial proper subgroup is cyclic of some fixed prime order. Clearly, such a group cannot contain $F_{\infty}$ as a subgroup, and it is Ol'shanskij's contribution that shows Tarski monster groups exist and are non-amenable.

On the other hand, every group with the weak Powers property, as defined in BN88, has a non-cyclic free subgroup. A proof can be found in [llH07, which also lists classes of groups that have the (weak) Powers property. We just mention that all free products $\Gamma_{1} * \Gamma_{2}$ with $\left|\Gamma_{1}\right| \geq 2,\left|\Gamma_{2}\right| \geq 3$ have the Powers property, and therefore Proposition 4.2 applies.

We may derive the following from Theorem 3.5 and Proposition 4.2.

Corollary 4.4. Let $A$ be a separable, unital $C^{*}$-algebra that contains a countable sequence of pairwise orthogonal, full elements (e.g., A is simple and non-elementary), and let $\Gamma$ be a group that contains a non-cyclic free subgroup. Then $A \otimes_{\max } C_{r}^{*}(\Gamma)$ is singly generated.

Example 4.5. Let $\Gamma_{1}, \Gamma_{2}$ be two groups that contain non-cyclic free subgroups. Then $C_{r}^{*}\left(\Gamma_{1} \times \Gamma_{2}\right) \cong C_{r}^{*}\left(\Gamma_{1}\right) \otimes_{\max } C_{r}^{*}\left(\Gamma_{2}\right)$ is singly generated. For example, for 
any $k, l \in\{2,3, \ldots, \infty\}$, the $C^{*}$-algebra $C_{r}^{*}\left(F_{k}\right) \otimes_{\max } C_{r}^{*}\left(F_{l}\right)$ is singly generated. In particular, $C_{r}^{*}\left(F_{\infty}\right) \otimes_{\max } C_{r}^{*}\left(F_{\infty}\right)$ is singly generated.

It was pointed out to the authors by S. Wassermann that $C_{r}^{*}\left(F_{k}\right) \otimes C_{r}^{*}\left(F_{l}\right)$ is not $\mathcal{Z}$-stable, for any $k, l \in\{2,3, \ldots, \infty\}$. In fact, if $C_{r}^{*}\left(F_{k}\right) \otimes C_{r}^{*}\left(F_{l}\right) \cong A \otimes B \otimes C$, then one of the three algebras $A, B$ or $C$ is isomorphic to $\mathbb{C}$. This is a generalization of the fact that $C_{r}^{*}\left(F_{k}\right)$ is tensorially prime, and it can be proved similarly.

We note that it is a difficult open problem whether $C_{r}^{*}\left(F_{k}\right)$ itself is singly generated.

Question 4.6. Given a non-amenable (discrete) group $\Gamma$, does $C_{r}^{*}(\Gamma)$ admit a unital embedding of $\mathcal{Z}$ ?

For each group $\Gamma$, the trivial group-morphism $\Gamma \rightarrow\{1\}$ induces a surjective morphism $C^{*}(\Gamma) \rightarrow \mathbb{C}$. Thus, the Jiang-Su algebra can never unitally embed into a full group $C^{*}$-algebra. If $\Gamma$ is amenable, then $C_{r}^{*}(\Gamma) \cong C^{*}(\Gamma)$, and consequently there is no unital embedding of $\mathcal{Z}$ into the reduced group $C^{*}$-algebra of an amenable group.

On the other hand, if $\Gamma$ contains a non-cyclic free subgroup, then Proposition 4.2 gives a positive answer to Question 4.6. As noted in Remark 4.3, not every nonamenable group contains a non-cyclic free subgroup. However, it is known that the reduced group $C^{*}$-algebra of a non-amenable group has no finite-dimensional representations, which is a necessary condition for the Jiang-Su algebra to embed.

\section{ACKNOWLEDGMENTs}

The first-named author thanks Mikael Rørdam for valuable comments, especially on the applications in Section 4

\section{REFERENCES}

[AP77] Charles A. Akemann and Gert K. Pedersen, Ideal perturbations of elements in $C^{*}$ algebras, Math. Scand. 41 (1977), no. 1, 117-139. MR0473848 (57 \#13507)

[Arv77] William Arveson, Notes on extensions of $C^{*}$-algebras, Duke Math. J. 44 (1977), no. 2, 329-355. MR0438137 (55 \#11056)

[BN88] Florin Boca and Viorel Niţică, Combinatorial properties of groups and simple $C^{*}$ algebras with a unique trace, J. Operator Theory 20 (1988), no. 1, 183-196. MR.972188 (90i:22007)

[Dad09] Marius Dadarlat, Continuous fields of $C^{*}$-algebras over finite dimensional spaces, Adv. Math. 222 (2009), no. 5, 1850-1881, DOI 10.1016/j.aim.2009.06.019. MR2555914(2010j:46102)

[DHTW09] Marius Dadarlat, Ilan Hirshberg, Andrew S. Toms, and Wilhelm Winter, The Jiang-Su algebra does not always embed, Math. Res. Lett. 16 (2009), no. 1, 23-26. MR2480557 (2010b:46130)

[dlH07] Pierre de la Harpe, On simplicity of reduced $C^{*}$-algebras of groups, Bull. Lond. Math. Soc. 39 (2007), no. 1, 1-26, DOI 10.1112/blms/bdl014. MR2303514(2008a:22004)

[DR98] K. J. Dykema and M. Rørdam, Projections in free product $C^{*}$-algebras, Geom. Funct. Anal. 8 (1998), no. 1, 1-16, DOI 10.1007/s000390050046. MR1601917 (99d:46075)

[DR00] Kenneth J. Dykema and Mikael Rørdam, Projections in free product $C^{*}$-algebras. II, Math. Z. 234 (2000), no. 1, 103-113, DOI 10.1007/s002090050505. MR.1759493 (2001f:46088)

[DSSW08] Ken Dykema, Allan Sinclair, Roger Smith, and Stuart White, Generators of $\mathrm{II}_{1}$ factors, Oper. Matrices 2 (2008), no. 4, 555-582, DOI 10.7153/oam-02-35. MR2468882 (2010c:46135) 
[ER06] George A. Elliott and Mikael Rørdam, Perturbation of Hausdorff moment sequences, and an application to the theory of $C^{*}$-algebras of real rank zero, Operator Algebras: The Abel Symposium 2004, Abel Symp., vol. 1, Springer, Berlin, 2006, pp. 97-115, DOI 10.1007/978-3-540-34197-0_5. MR2265045(2007m:46085)

[ET08] George A. Elliott and Andrew S. Toms, Regularity properties in the classification program for separable amenable $C^{*}$-algebras, Bull. Amer. Math. Soc. (N.S.) 45 (2008), no. 2, 229-245, DOI 10.1090/S0273-0979-08-01199-3. MR2383304 (2009k:46111)

[FTT11] I. Farah, A. Tornquist, and A.S. Toms, Turbulence, orbit equivalence, and the classification of nuclear $C^{*}$-algebras, preprint 2011, to appear in J. Reine Angew. Math.

[Ge03] Li Ming Ge, On "Problems on von Neumann algebras by R. Kadison, 1967", Acta Math. Sin. (Engl. Ser.) 19 (2003), no. 3, 619-624, DOI 10.1007/s10114-003-0279-x. With a previously unpublished manuscript by Kadison; International Workshop on Operator Algebra and Operator Theory (Linfen, 2001). MR2014042(2005a:46120)

[GP98] Liming Ge and Sorin Popa, On some decomposition properties for factors of type $\mathrm{II}_{1}$, Duke Math. J. 94 (1998), no. 1, 79-101, DOI 10.1215/S0012-7094-98-09405-4. MR.1635904 (99j:46070)

[JS99] Xinhui Jiang and Hongbing Su, On a simple unital projectionless $C^{*}$-algebra, Amer. J. Math. 121 (1999), no. 2, 359-413. MR.1680321 (2000a:46104)

[JW11] M. Johanesova and W. Winter, The similarity problem for $\mathcal{Z}$-stable $C^{*}$-algebras, Bull. Lond. Math. Soc. 44 (2012), no. 6, 1215-1220. MR.3007654

[Kad67] R. Kadison, Problems on von Neumann algebras, unpublished manuscript, presented at Conference on Operator Algebras and Their Applications, Louisiana State Univ., Baton Rouge, La., 1967.

[Kas88] G. G. Kasparov, Equivariant KK-theory and the Novikov conjecture, Invent. Math. 91 (1988), no. 1, 147-201, DOI 10.1007/BF01404917. MR918241 (88j:58123)

[Lon84] R. Longo, Solution of the factorial Stone-Weierstrass conjecture. An application of the theory of standard split $W^{*}$-inclusions, Invent. Math. 76 (1984), no. 1, 145-155, DOI 10.1007/BF01388497. MR739630(85m:46057a)

[LS10] W. Li and J. Shen, A note on approximately divisible $C^{*}$-algebras, preprint, arXiv:0804.0465, 2010.

[Nag04] Masaru Nagisa, Single generation and rank of $C^{*}$-algebras, Operator algebras and applications, Adv. Stud. Pure Math., vol. 38, Math. Soc. Japan, Tokyo, 2004, pp. 135143. MR2059805 (2005a:46110)

[OZ76] Catherine L. Olsen and William R. Zame, Some $C^{*}$-alegebras with a single generator, Trans. Amer. Math. Soc. 215 (1976), 205-217. MR.0388114 (52 \#8951)

[Pea62] Carl Pearcy, $W^{*}$-algebras with a single generator, Proc. Amer. Math. Soc. 13 (1962), 831-832. MR0152904 (27 \#2875)

[Pop84] S. Popa, Semiregular maximal abelian ${ }^{*}$-subalgebras and the solution to the factor state Stone-Weierstrass problem, Invent. Math. 76 (1984), no. 1, 157-161, DOI 10.1007/BF01388498. MR739631 (85m:46057b)

[Rob12] Leonel Robert, Classification of inductive limits of 1-dimensional NCCW complexes, Adv. Math. 231 (2012), no. 5, 2802-2836, DOI 10.1016/j.aim.2012.07.010. MR 2970466

[Rør04] Mikael Rørdam, The stable and the real rank of $\mathcal{Z}$-absorbing $C^{*}$-algebras, Internat. J. Math. 15 (2004), no. 10, 1065-1084, DOI 10.1142/S0129167X04002661. MR2106263 (2005k:46164)

[RW10] Mikael Rørdam and Wilhelm Winter, The Jiang-Su algebra revisited, J. Reine Angew. Math. 642 (2010), 129-155, DOI 10.1515/CRELLE.2010.039. MR2658184 (2011i:46074)

[She09] David Sherman, On cardinal invariants and generators for von Neumann algebras, Canad. J. Math. 64 (2012), no. 2, 455-480, DOI 10.4153/CJM-2011-048-2. MR2953208

[She10] David Sherman, Locally inner automorphisms of operator algebras, Q. J. Math. 61 (2010), no. 2, 241-254, DOI 10.1093/qmath/han039. MR2646087 (2011g:46114)

[Top68] David M. Topping, UHF algebras are singly generated, Math. Scand. 22 (1968), 224226 (1969). MR0244783 (39 \#6097)

[TW08] Andrew S. Toms and Wilhelm Winter, Z्Z-stable ASH algebras, Canad. J. Math. 60 (2008), no. 3, 703-720, DOI 10.4153/CJM-2008-031-6. MR2414961 (2009m:46089) 
[vN31] J. von Neumann, Über Funktionen von Funktionaloperatoren (German), Ann. of Math. (2) 32 (1931), no. 2, 191-226, DOI 10.2307/1968185. MR.1502991

[Wil74] Paul Willig, Generators and direct integral decompositions of $W^{*}$-algebras, Tôhoku Math. J. (2) 26 (1974), 35-37. MR0336369 (49 \#1144)

[Win07] W. Winter, Localizing the Elliott conjecture at strongly self-absorbing $C^{*}$-algebras, preprint 2007, to appear in J. Reine Angew. Math.

[Win11] Wilhelm Winter, Strongly self-absorbing $C^{*}$-algebras are $\mathcal{Z}$-stable, J. Noncommut. Geom. 5 (2011), no. 2, 253-264, DOI 10.4171/JNCG/74. MR2784504 (2012e:46132)

[Win12] Wilhelm Winter, Nuclear dimension and $\mathcal{Z}$-stability of pure $\mathrm{C}^{*}$-algebras, Invent. Math. 187 (2012), no. 2, 259-342, DOI 10.1007/s00222-011-0334-7. MR2885621

[Wog69] Warren Wogen, On generators for von Neumann algebras, Bull. Amer. Math. Soc. 75 (1969), 95-99. MR0236725 (38 \#5020)

Mathematisches Institut Der Universität Münster, Einsteinstr. 62, 48149 Münster, GERMANY

E-mail address: hannes.thiel@uni-muenster.de

Mathematisches Institut der Universität Münster, Einsteinstr. 62, 48149 Münster, GERMANY

E-mail address: wwinter@uni-muenster.de 